\title{
UTILISATION D'UN CLUB DE LECTURE COMME MÉTHODE PÉDAGOGIQUE POUR FAVORISER L'APPROPRIATION DES DONNÉES PROBANTES PAR DES ÉTUDIANTES AU BACCALAURÉAT EN SCIENCES INFIRMIÈRES
}

Maud-Christine Chouinard, Mélissa Lavoie, Marie-Ėve Poitras, Maxime Sasseville, Louise Catherine de Jordy, Ariane Girard

\section{Association de recherche en soins infirmiers (ARSI) | « Recherche en soins infirmiers »}

2015/1 N 120 | pages 35 à 46

ISSN 0297-2964

Article disponible en ligne à l'adresse :

http://www.cairn.info/revue-recherche-en-soins-infirmiers-2015-1-page-35.htm

\section{!Pour citer cet article :}

Maud-Christine Chouinard et al., «Utilisation d'un club de lecture comme méthode pédagogique pour favoriser l'appropriation des données probantes par des étudiantes au baccalauréat en sciences infirmières ", Recherche en soins infirmiers 2015/1 ( $\left.{ }^{\circ} 120\right)$, p. 35-46. DOI 10.3917/rsi.120.0035

Distribution électronique Cairn.info pour Association de recherche en soins infirmiers (ARSI).

(C) Association de recherche en soins infirmiers (ARSI). Tous droits réservés pour tous pays.

La reproduction ou représentation de cet article, notamment par photocopie, n'est autorisée que dans les limites des conditions générales d'utilisation du site ou, le cas échéant, des conditions générales de la licence souscrite par votre établissement. Toute autre reproduction ou représentation, en tout ou partie, sous quelque forme et de quelque manière que ce soit, est interdite sauf accord préalable et écrit de l'éditeur, en dehors des cas prévus par la législation en vigueur en France. Il est précisé que son stockage dans une base de données est également interdit. 


\section{Utilisation d'un club de lecture comme méthode pédagogique pour favoriser l'appropriation des données probantes par des étudiantes au baccalauréat en sciences infirmières}

\section{The journal club as a teaching strategy to develop evidence-based practice for undergraduate students in nursing}

\section{Maud-Christine CHOUINARD}

Infirmière, Ph.D., Professeure agrégée, Université du Québec à Chicoutimi, Canada

\section{Mélissa LAVOIE}

Infirmière, MSc., Ph.D.(c), Professeure adjoint, Université du Québec à Chicoutimi, Canada

\section{Marie-Ève POITRAS}

Infirmière, MSc., Ph.D.(c), Chargée de cours, Université du Québec à Chicoutimi, Canada

\section{Maxime SASSEVILLE}

Infirmier, MSc., Ph.D.(c), Chargé de cours, Université du Québec à Chicoutimi, Canada

\section{Louise Catherine DE JORDY}

Infirmière, BSc., MSc(c), Étudiante à la maîtrise en sciences infirmières, Université du Québec à Chicoutimi, Canada

\section{Ariane GIRARD}

Infirmière, $\mathrm{BSc}$., $\mathrm{MSc}(\mathrm{c})$, Étudiante à la maitrise en sciences infirmières, Université du Québec à Chicoutimi

\section{Remerciements}

Les auteurs désirent remercier les personnes impliquées comme responsables de l'animation des sous-groupes, notamment Chantale Thériault, étudiante infirmière praticienne spécialisée ; Isabelle Dufour, étudiante à la maîtrise en sciences infirmières ; Suzanne Aucoin, professeure retraitée en sciences infirmières ; Nicole Lessard et Jérémy Allard, assistants de recherche.

Les auteurs souhaitent également remercier l'équipe de professeures du module des sciences infirmières et de la santé, le directeur du département des sciences de la santé, monsieur Étienne Hébert, et la doyenne des études de premier cycle, madame Carole Dion, pour leur appui au projet et leur soutien financier.

Ils souhaitent également souligner l'accompagnement offert aux étudiantes par madame Caroline Bellemare, étudiante au programme de maîtrise en anglais langue seconde, dans la lecture d'article en anglais.

Pour citer l'article :

Chouinard MC, Lavoie M, Poitras ME, Sasseville M, de Jordy LC, Girard A. Utilisation d'un club de lecture comme méthode pédagogique pour favoriser l'appropriation des données probantes par des étudiantes au baccalauréat en sciences infirmières. Rech Soins Infirm, 2015 mar;(I20):35-46. 


\begin{abstract}
RÉS U M É
Introduction : la pratique infirmière contemporaine nécessite une mise à jour continue des connaissances, notamment à partir des résultats de recherche publiés dans des articles scientifiques. Contexte : comme exigence du curriculum universitaire en sciences infirmières, les étudiantes doivent développer leurs compétences en regard de l'évaluation des évidences de recherche. Objectifs : l'objectif de cette étude était d'explorer l'appréciation et les effets d'une nouvelle approche pédagogique, le club de lecture, auprès d'étudiantes de baccalauréat en sciences infirmières dans le cadre d'un cours de recherche. Méthode : un dispositif qualitatif descriptif exploratoire a été utilisé. Un questionnaire à questions ouvertes a été complété par des étudiantes $(n=4 I)$ suite à la participation au club de lecture à l'intérieur du cours de recherche en sciences infirmières afin d'obtenir leur appréciation et leur perception des effets de cette méthode pédagogique. Résultats : la participation aux clubs de lecture apparaît avoir : I) favorisé l'initiation à l'univers scientifique, 2) amélioré plusieurs compétences en lien avec l'évaluation d'articles scientifiques et 3) augmenté la motivation en regard de la pratique basée sur les données probantes. Conclusion : cette nouvelle méthode pédagogique utilisée à l'intérieur du cours de recherche s'est avérée pertinente et a grandement suscité l'intérêt des étudiantes en sciences infirmières.
\end{abstract}

Mots clés : enseignement universitaire, recherche, sciences infirmières, club de lecture, données probantes.

A B S T R A C T

Introduction : contemporary nursing practice requires constant updating of knowledge, especially in regards to research results found in academic or scientific papers. Context : as part of the university curriculum in nursing, students must develop their skills in relation to the understanding and evaluation of research evidence. Objectives : the objective of this study was to explore the appreciation and effects of a new teaching approach, the use of a journal club within a nursing research course, with undergraduate students in nursing sciences. Method : as part of a qualitative exploratory descriptive design, a questionnaire with open-ended questions about their appreciation and perception of the effects of the journal club was administered to the participating students $(n=4 I)$ at the end of the course. Results : participation in the journal club appears to have provided an introduction to the scientific area of nursing, to have improved several skills related to the evaluation of scientific articles and has increased motivation to adopt evidence-based practice. Conclusion : the use of a journal club within an undergraduate research course in nursing was a relevant teaching method that aroused great interest in the undergraduate students in nursing.

Key words : university teaching, research, nursing sciences, journal club, evidence-based.

\section{INTRODUCTION}

La pratique infirmière contemporaine nécessite une mise à jour continue des connaissances, notamment à partir des résultats de recherche publiés dans des articles scientifiques. La pratique infirmière basée sur les données probantes (evidence-based nursing) est l'utilisation consciencieuse, explicite et judicieuse d'informations en provenance de recherches scientifiques et basées sur des théories, permettant de prendre des décisions sur les soins à dispenser en tenant compte des ressources disponibles ainsi que des besoins et des préférences des patients (I). Le transfert des connaissances issues de la recherche dans la pratique est un élément essentiel pour veiller à ce que les soins aux patients soient basés sur des principes scientifiques (2).

À l'ère de la pratique infirmière basée sur les données probantes, il est attendu que les étudiantes finissant leur formation au niveau du baccalauréat en sciences infirmières soient capables d'identifier les écrits scientifiques pertinents en lien avec un problème clinique et de juger de la pertinence des évidences scientifiques qui y sont décrites ainsi que de leur qualité, en vue de recommander ou non l'utilisation et l'inclusion de ces évidences dans leur milieu de pratique clinique $(3,4)$. L'obtention d'un baccalauréat en sciences infirmières n'est que le début du processus d'apprentissage de données probantes, continuellement renouvelées lors de la carrière d'une infirmière, permettant ainsi une pratique compétente et sécuritaire modulée, en partie, par l'apport des recherches scientifiques $(5,6)$. Cela est d'autant plus important dans le contexte québécois actuel où la norme d'entrée dans la profession infirmière après la formation universitaire seulement fait l'objet d'un débat important (7). D'une part, l'importance du jugement clinique appuyée sur les évidences scientifiques est reconnue dans le cadre législatif. D'autre part, la recherche et les données probantes en sciences infirmières ne sont pas enseignées dans la formation de niveau collégial en soins infirmiers.

Historiquement, les étudiantes' en soins infirmiers ont souvent questionné la valeur du cours de recherche en sciences infirmières dans leur cursus de premier cycle et ne semblent pas en mesure de déceler l'application clinique des évidences de recherche (8). Ce sont des préoccupations importantes à la lumière de la priorité croissante mise sur la pratique infirmière fondée sur les données probantes. Les

\footnotetext{
' Lire partout étudiants/étudiantes, infirmiers/infirmières, participants/ participantes.
} 


\section{Utilisation d'un club de lecture comme méthode pédagogique pour favoriser l'appropriation des données probantes par des étudiantes au baccalauréat en sciences infirmières}

infirmières enseignantes ont souvent du mal à élaborer des méthodes pédagogiques engageantes et significatives du point de vue des étudiantes de premier cycle en sciences infirmières (9). Certains auteurs soutiennent que des approches novatrices pour l'enseignement de la recherche en sciences infirmières au premier cycle doivent être mises en place pour favoriser les apprentissages et l'engagement des étudiantes de la génération $\mathrm{Y}$, qui constituent la plus importante proportion des étudiantes actuellement inscrites au niveau du baccalauréat en sciences infirmières au Québec. Ils suggèrent notamment de favoriser les apprentissages expérientiels de groupe. Il est également suggéré par des auteurs de voir les étudiantes comme des agents catalyseurs pouvant favoriser le transfert de connaissances vers les milieux de pratique (I0).

Parmi les méthodes utilisées dans les milieux cliniques afin de favoriser l'appropriation des données probantes en sciences infirmières, à l'instar d'autres disciplines du domaine de la santé, l'approche du club de lecture est retrouvée (I I). Alors que de nombreux articles scientifiques en sciences infirmières expliquent la logistique de la création et de l'utilisation d'un club de lecture dans des contextes cliniques, peu d'articles décrivant l'évaluation de l'utilisation de cette approche comme une méthode pédagogique pertinente dans l'enseignement des sciences infirmières ont été répertoriés (12). Les quelques articles retrouvés proviennent tous des États-Unis et rapportent souvent l'utilisation de clubs de lecture en dehors des activités académiques formelles $(12,13,14,4)$, s'appliquant ainsi peu à la réalité des étudiantes au niveau du baccalauréat en sciences infirmières au Québec.

L'objectif de cette étude était d'explorer l'appréciation et les effets d'une nouvelle approche pédagogique, le club de lecture, auprès d'étudiantes de baccalauréat en sciences infirmières dans le cadre d'un cours de recherche.

\section{CADRE DE RÉFÉRENCE}

Ce cadre de référence apporte premièrement une définition d'un club de lecture. II s'ensuit une description brève des diverses études effectuées jusqu'à maintenant concernant son utilisation et ses effets en contexte d'enseignement universitaire en sciences infirmières.

\section{Définition des clubs de lecture}

L'utilisation des clubs de lecture est largement reconnue dans les domaines de la formation et de la pratique médicale $(2,15)$. Dans la discipline infirmière, bien que plusieurs exemples de son utilisation dans les milieux de pratique sont retrouvés, très peu d'articles décrivant son utilisation et ses effets en contexte de formation universitaire sont présents dans la littérature (12). En effet, il semble que très peu de milieux d'enseignement universitaire l'aient adopté à ce jour comme méthode d'enseignement, même si l'utilisation d'un club de lecture semble favoriser chez les étudiants un apprentissage participatif en leur donnant un moyen de mettre à jour leurs connaissances sur une base continue, de critiquer les articles scientifiques et d'envisager une pratique future basée sur les données probantes (2). Les études sur l'efficacité des clubs de lecture dans le milieu universitaire se retrouvent principalement dans la littérature médicale et, bien que les avantages sont susceptibles d'être similaires chez les étudiantes en sciences infirmières, les effets de cette approche restent à démontrer chez cette clientèle étudiante (I2). Quelques auteurs ont suggéré que le club de lecture devrait être introduit comme méthode pédagogique dans les cours du niveau de baccalauréat, notamment dans les cours d'initiation à la recherche $(4,16)$.

En milieu de pratique, un club de lecture se définit comme étant « des rencontres au cours desquelles des participants discutent d'articles scientifiques ou professionnels abordant des sujets en lien avec leur pratique professionnelle. ॥ (I7). Lorsqu'utilisé en milieu académique, le but d'un club de lecture est d'améliorer les connaissances des étudiants par rapport au processus de la recherche, de mettre en pratique leurs compétences dans l'évaluation d'articles scientifiques dans un environnement de soutien, et d'évaluer les résultats de la recherche pour une utilisation dans la pratique des soins infirmiers (12).

\section{Utilisation d'un club de lecture dans la formation universitaire en sciences infirmières}

Seulement sept articles relatant l'utilisation d'un club de lecture dans la formation universitaire de premier cycle en sciences infirmières ont été retrouvés. Dans la majorité des cas, les clubs de lecture étaient réalisés en dehors des activités académiques créditées $(4,18,19,20)$. Dans ces derniers, les clubs de lecture ont pris différentes formes. En contexte canadien, la réalisation du club de lecture par des étudiantes au baccalauréat en sciences infirmières $(n=35$ étudiantes de la première à la dernière année d'études) a été effectuée via la plateforme WebCT (4). Il a été constitué d'au moins quatre échanges planifiés autour d'articles choisis par les chercheurs. Un autre article décrit l'utilisation de clubs de lecture comme activité post-stage pour des étudiantes en début de programme (18).

Les trois autres articles ont porté sur des projets de clubs de lecture effectués conjointement avec le milieu clinique. Le premier décrit un vaste projet de recherche (200 infirmières des milieux cliniques et 200 étudiantes) impliquant une collaboration entre le milieu universitaire et divers milieux de pratique (soins prolongés, santé mentale, médecine et chirurgie) (19). Le deuxième décrit un projet similaire sans entrer dans les détails de l'évaluation faite (20). Le troisième a été implanté dans diverses unités de soins d'un hôpital universitaire sur une période de 
quatre années (2007-20II) (2I). Dans ces trois projets, les questions étudiées dans les clubs de lecture émanaient des milieux cliniques, la démarche d'analyse critique était principalement effectuée par les étudiantes et les clubs de lecture étaient réalisés en milieu clinique. Dans un des projets, des étudiantes graduées ont été impliquées pour servir de modèle de rôle (20).

Seulement deux articles ont décrit l'utilisation de clubs de lecture dans le cadre de cours universitaires crédités. Le premier décrit une étude pilote avec des étudiantes de $2^{\text {ème }}$ année $(\mathrm{n}=62)$ dans le cadre d'un cours sur les maladies chroniques (22). L'autre étude constitue la seule étude ayant évalué l'utilisation d'un club de lecture dans le cadre d'un cours universitaire de recherche en sciences infirmières (I2). Dans cette étude, les étudiantes d'un cours de recherche au baccalauréat en sciences infirmières ont été invitées à choisir différentes stratégies pédagogiques parmi lesquelles se trouvaient des clubs de lecture. L'évaluation du cours comportait des questions spécifiques sur l'appréciation des clubs de lecture par les étudiantes.

Dans trois de ces six études, les auteurs décrivent avoir utilisé des feuilles ou grilles d'analyse critique d'écrits scientifiques pour guider les étudiantes dans la préparation et la réalisation des clubs de lecture $(4,18,22)$. Dans les six études, les résultats ont été mesurés principalement à l'aide de questionnaires développés par les auteurs. Seul l'article de Steenbeck rapporte avoir également analysé les feuilles de travail des étudiantes pour établir si les étudiantes s'étaient améliorées progressivement au cours des quatre clubs de lecture auxquelles elles ont assisté (4).

L'ensemble de ces études rapportent une appréciation positive des participantes bien que certaines d'entre elles décrivent très peu la méthodologie d'évaluation utilisée pour obtenir leurs résultats $(20,22)$. Les principaux effets décrits concernaient : I) une meilleure compréhension des processus de la recherche scientifique $(12) ; 2$ ) une amélioration des compétences d'analyse critique d'articles scientifiques $(12,22) ; 3)$ une meilleure compréhension de ce que peuvent leur apporter les données probantes $(12,4)$; 4) une formule encourageant les interactions et le dialogue entre les participants (4); et 5) un soutien à une utilisation ultérieure des résultats de recherche $(2,16,20)$.

Très peu relatent des obstacles, barrières ou limites à la méthode des clubs de lecture. Parmi ceux-ci, Laaksonen et al. ont décrit des problèmes d'assiduité des étudiantes, de valorisation des nouvelles compétences acquises en analyse critique des articles de recherche et de pertinence des clubs de lecture lorsqu'un trop petit nombre (2) ou un trop grand nombre de personnes y participent (19). Les deux barrières les plus fréquemment décrites dans l'étude de Steenbeck et al. par les participants ont été leur inhabilité à trouver des évidences scientifiques et la difficulté de bien les comprendre
(4). En général, les barrières sont l'hésitation des infirmières à lire des articles scientifiques, leur incertitude sur comment bien les analyser et les interpréter (2).

Bien que ces écrits soulèvent la pertinence de l'utilisation d'un club de lecture, le manque de connaissances sur les effets de son utilisation dans le cadre d'un cours de recherche au baccalauréat en sciences infirmières et sur les processus pouvant expliquer ces effets appellent à une poursuite de cette démonstration.

\section{MÉTHODOLOGIE}

\section{Population étudiée}

La population cible étudiée était constituée des étudiantes inscrites dans un programme de baccalauréat en sciences infirmières dans une université québécoise. Le contexte de la formation universitaire en sciences infirmières au Québec est caractérisé par la présence de plusieurs cheminements de formation, impliquant une diversité de populations étudiantes fréquentant les établissements universitaires. Le premier cheminement (formation intégrée, 2 ans) est celui d'étudiantes ayant complété une formation de niveau collégial en soins infirmiers depuis moins de cinq ans et possédant ainsi déjà le titre d'infirmière. Le deuxième (formation de perfectionnement, 3 ans) s'adresse aux infirmières qui ont terminé leur formation de niveau collégial en soins infirmiers il $y$ a plus de cinq ans. Le troisième cheminement (formation initiale, 3 ans) s'adresse aux étudiantes admises avec une formation collégiale générale ou dans un domaine autre.

Récemment, l'Ordre des infirmières et infirmiers du Québec (OIIQ) a statué que la pratique fondée sur les données probantes est une compétence acquise par la formation en recherche telle que dispensée dans les programmes de baccalauréat en sciences infirmières des universités québécoises (7). Il est à noter que tous les programmes de baccalauréat en sciences infirmières au Québec comportent un cours d'introduction à la recherche en sciences infirmières.

En 20I3, 3041 permis d'exercice (86\% pour des femmes) ont été délivrés par l'OIIQ (23). De ce nombre, $70 \%$ sont titulaires d'un diplôme d'études collégial. Parmi cette relève, $47 \%$ prendront la décision de poursuivre la formation universitaire (formation intégrée).

La population accessible pour cette étude a été constituée des étudiantes inscrites au cours d'initiation à la recherche à I'Université du Québec à Chicoutimi (UQAC). Le seul critère d'inclusion à l'étude était d'être une étudiante inscrite au cours "Processus de recherche en sciences infirmières" (SSOI222) de I'UQAC au trimestre automne 2012. II n'y a eu aucun critère d'exclusion à l'étude prévu. 


\section{Utilisation d'un club de lecture comme méthode pédagogique pour favoriser l'appropriation des données probantes par des étudiantes au baccalauréat en sciences infirmières}

\section{Devis}

L'étude a été développée selon un devis qualitatif descriptif exploratoire. Le recours à cette méthode permet d'explorer et de décrire un phénomène encore peu étudié à ce jour selon une population définie. L'utilisation de ce type de devis amènera à la découverte de nouvelles évidences afin d'offrir les assises théoriques de futurs projets de recherche (24).

\section{Description de l'intervention}

L'intervention s'est déroulée au cours d'un trimestre universitaire de 4 mois. Tout au long de cette période, les étudiantes participantes, de même que les autres, ont reçu un enseignement sur la recherche scientifique et les données probantes en sciences infirmières avec une méthode pédagogique sous forme d'un club de lecture. Cette méthode pédagogique a été approuvée par la direction du module des sciences infirmières et de la santé et l'équipe de professeures de l'Université du Québec à Chicoutimi.

La structure du cours, étalée sur 15 semaines, a été réalisée de la manière suivante. Les cinq premières séances de cours ( 15 heures) ont été consacrées à un enseignement magistral sur : I) les notions de base en recherche en regard des données probantes, 2) les différents devis de recherche et 3 ) l'analyse critique d'articles scientifiques avec des exemples. Au cours des semaines de cours théoriques, les étudiantes ont eu à développer individuellement une question PICO : patient ou population $(P)$; intervention ou domaine d'intérêt (issue of interest) (I) ; intervention de comparaison (C) ; résultat(s) visé(s) (outcome(s) of interest) (O) (25). Les étudiantes ont été invitées à formuler cette question à partir d'une observation ou d'une question clinique provenant de leur expérience dans leur domaine de pratique. Par la suite, elles devaient trouver dans les banques de données (principalement CINAHL et Medline) huit articles scientifiques apportant une réponse à cette question ${ }^{2}$. Un exemple d'une question d'un étudiant a été : « Pour les patients intubés hospitalisés dans une unité de soins intensifs $(P)$, quels soins de bouche doivent être apportés (I), en comparaison avec l'absence de soins de bouche $(C)$ pour diminuer les risques d'infections nosocomiales $(\mathrm{O})$ ? ॥

Les huit séances suivantes ont été organisées sous la forme de club de lecture en sous-groupes d'environ 10 participantes en fonction de leur champ de pratique d'intérêt. Six sousgroupes ont ainsi été formés : en soins critiques (incluant les milieux de l'urgence et des soins intensifs), en gériatrie et

\footnotetext{
2 À l'UQAC, les étudiantes de tous les programmes de baccalauréat en sciences infirmières doivent suivre une formation sur les compétences informationnelles d'une durée de 4 heures dans le cadre d'un cours intitulé «Introduction aux programmes en sciences infirmières ». Cette formation vise à les habiliter à développer une stratégie de recherche documentaire et à l'appliquer dans le cadre d'une recherche documentaire dans les banques de données.
}

soins de longue durée, en santé mentale, en santé maternelle et infantile, et en médecine-chirurgie (deux sous-groupes). Chacun des sous-groupes était animé par un responsable (étudiantes graduées en sciences infirmières, assistantes de recherche ou professeurs). Ainsi, à quatre reprises au cours de la session, selon un horaire établi à l'avance, chaque étudiante a présenté devant ses pairs ayant un même domaine d'intérêt, une analyse critique d'un des articles scientifiques pertinents en lien avec sa question de recherche. Cette présentation devait durer 15 minutes, suivie d'une période de questions de 5 à 10 minutes à l'intérieur de laquelle les autres étudiantes et l'animatrice du sous-groupe devaient poser des questions ou faire des commentaires. L'analyse critique de chaque article à devis quantitatif ou qualitatif a été basée sur des grilles d'analyse critique adaptées des feuilles de travail et d'accompagnement du lecteur de Davies \& Logan (26). En plus d'aborder les éléments de critique d'articles scientifiques traditionnels, ces feuilles de travail contiennent plusieurs éléments d'évaluation se rapportant à l'applicabilité clinique des résultats pour que l'étudiante se questionne à ce niveau. En ce qui concerne les méta-analyses et les revues de littérature, deux autres grilles ont été adaptées de Fortin, Hudon et Poitras (27) et proposées aux étudiantes. À la fin de chaque club de lecture, les étudiantes devaient remettre leur grille complétée pour évaluation par la professeure. Les deux derniers cours ont été consacrés à un retour sur la matière du cours.

Étant donné que la langue de communication scientifique privilégiée est actuellement l'anglais et que la grande partie des résultats de recherche en sciences infirmières sont publiés dans cette langue (28), la professeure responsable s'est assurée de pouvoir offrir du soutien à la traduction aux étudiantes via une subvention institutionnelle dans le cadre d'un programme visant la réussite académique de l'Université du Québec à Chicoutimi. Ce soutien a consisté à embaucher une étudiante de $2^{\text {ème }}$ cycle en anglais langue seconde de l'Université pour qu'elle accompagne les étudiantes s'initiant à la lecture d'écrits scientifiques en anglais. Le soutien apporté a pris la forme de stratégies visant la compréhension adéquate des articles en mettant la priorité sur le vocabulaire scientifique commun entre le français et l'anglais, ainsi que sur la structure d'un article scientifique afin de favoriser le repérage de l'information.

\section{Outils de collecte des données}

Les données concernant l'appréciation et les effets de la participation aux clubs de lecture ont été obtenues à partir d'un questionnaire avec questions ouvertes développé par les chercheurs. Les questions posées étaient : I) Qu'est-ce que votre participation aux clubs de lecture pendant la session vous a apporté par rapport à la lecture d'articles scientifiques? 2) Comment les clubs de lecture vous ont-ils aidé à mieux comprendre les écrits scientifiques ? 3) Quel est l'élément le plus important que vous retenez de l'analyse critique des 
écrits scientifiques ? 4) Comment pensez-vous appliquer les notions apprises dans votre pratique clinique dans l'avenir ? 5) Selon vous, quels éléments du fonctionnement des clubs de lecture seraient à améliorer ?

Des informations sociodémographiques ont été recueillies auprès des participantes afin d'établir leur profil général. Ce questionnaire comprenait des questions sur l'âge, le sexe, le programme d'études, le nombre de crédits universitaires obtenus jusqu'à maintenant, le statut d'étudiant (temps complet ou temps partiel), l'année d'obtention du permis d'exercice de la profession infirmière, le statut d'emploi (temps complet ou temps partiel), la spécialité professionnelle, si la personne a déjà effectué des études universitaires autres et si elle a déjà suivi une formation en lien avec les données probantes antérieurement.

\section{Déroulement de la collecte des données}

À la première séance de cours, les étudiantes inscrites au cours "Processus de recherche en sciences infirmières" (SSOI222) ont reçu de l'information sur l'étude et ont été invitées à y participer. Pour l'équipe de recherche, il semblait très intéressant pour illustrer un processus de recherche aux étudiantes, de leur faire vivre l'expérience d'être une participante à un projet de recherche. Les étudiantes intéressées ont alors été invitées à signer un formulaire de consentement. Tout le processus d'information et de recrutement a été fait par une assistante de recherche, en l'absence de la professeure du cours, pour assurer une participation libre et la confidentialité des données.

Pendant huit semaines, elles ont eu un enseignement sous la forme d'un club de lecture tel que décrit précédemment. À la dernière séance de cours, les étudiantes présentes ont complété le questionnaire évaluatif ainsi que le questionnaire sociodémographique.

\section{Considérations éthiques}

Cette étude a été approuvée par le Comité d'éthique de la recherche de l'Université du Québec à Chicoutimi (Québec, Canada). Tous les participants ont été invités à prendre connaissance et à signer un formulaire de consentement. Pour assurer la confidentialité des participantes, le recrutement de la population et la passation du questionnaire ont été réalisés en l'absence de la professeure par des assistantes de recherche.

\section{Analyse des données}

Les réponses à chaque question du questionnaire ont été retranscrites. Ce matériel écrit a fait l'objet d'une analyse de contenu et un co-codage a été effectué par trois chercheures de l'équipe (ML, MCC, MEP) afin de ressortir les concepts émergents en lien avec la participation à un club de lecture (29).

\section{RÉSULTATS ET DISCUSSIONS}

Cette partie présente premièrement les caractéristiques des participantes. Par la suite, les résultats obtenus à chacune des questions du questionnaire sont présentés et discutés dans l'ordre.

\section{Description de la population répondante}

Initialement, 55 étudiantes sur une possibilité de 58 ont accepté de prendre part à l'étude. Cependant, en raison de l'organisation du cours, seulement 4 I d'entre elles ont complété le questionnaire à la dernière séance (taux de participation de 7l \%).

L'échantillon final considéré dans l'étude est donc formé de $4 \mathrm{I}$ participantes (7 \% d'hommes), principalement de formation intégrée (87\%) à temps complet (97\%). Une grande proportion d'entre elles avaient une pratique clinique active $(68 \%)$ dans différents milieux de pratique en soins infirmiers, principalement en milieu hospitalier.

\section{Question I. Apport de la participation aux clubs de lecture}

II semble que la participation aux clubs de lecture ait été appréciée par plusieurs étudiantes telles que le confirment les extraits suivants : « Mais oui j'ai appris probablement plus que s'il n'y avait pas eu de club de lecture. Je ne connaissais rien à la recherche, maintenant je connais la base. » [.C ] $]^{3}$ « Continuez ce style. Cela nous fait travailler et mieux comprendre par nousmêmes. ॥ [.G]. « Cours à répéter à l'avenir. Ne pas revenir à l'ancien cours ! S.V.P. » [BB]. « Très pertinent comme méthode de travail, j'ai beaucoup appris ! " [AA]. Ces observations sont similaires avec celles faites dans les études sur l'utilisation de clubs de lecture en milieu universitaire $(12,22)$.

De plus la participation aux clubs de lecture a été riche pour les étudiantes à l'égard de deux domaines précis, soit l'initiation à l'univers scientifique et l'amélioration de compétences dans ce domaine.

\section{Initiation à l'univers scientifique}

Tout d'abord, la participation s'est avérée être une initiation à l'univers scientifique dans la discipline infirmière. Deux participantes se sont exprimées ainsi à ce sujet : " Cette participation au club de lecture m'a permis de me familiariser un peu avec des activités scientifiques. » [.B]. « Avant, je ne savais même pas que ça [les données probantes] existait. »[BF].

Également, le club de lecture a permis aux étudiantes de prendre conscience de l'importance de la recherche pour la

\footnotetext{
${ }^{3}$ Chaque extrait est suivi d'un code correspondant au questionnaire.
} 


\section{Utilisation d'un club de lecture comme méthode pédagogique pour favoriser l'appropriation des données probantes par des étudiantes au baccalauréat en sciences infirmières}

profession infirmière, plus particulièrement pour le domaine clinique. Les extraits de questionnaires suivants en témoignent : « Je me suis rendue compte que la recherche est importante dans notre profession. » [CA]. «Qu'elle [la recherche en sciences infirmières] nous permet d'avancer, de changer nos attitudes et nos méthodes de travail en prenant compte des données probantes. » [BE]. « J'ai compris en quoi les données probantes pouvaient me servir concrètement. » [BG].

Cette prise de conscience va dans le même sens que le Conseil international des infirmières (CII) qui avance que la recherche en sciences infirmières est indispensable pour I) générer de nouvelles connaissances, 2) faire progresser les sciences infirmières, 3) évaluer les pratiques et les services existants et finalement 4) apporter des informations qui serviront de repère pour l'enseignement en sciences infirmières, la pratique professionnelle, la recherche et la gestion dans ce domaine (30).

\section{Acquisition de compétences ayant trait au domaine scientifique}

La participation aux clubs de lecture a manifestement permis d'améliorer l'efficacité personnelle des étudiantes en regard de leur capacité à juger de la qualité et de la pertinence d'un article scientifique, ainsi que de l'utilité des résultats pour la pratique. Une participante s'est exprimée ainsi : " J'ai une plus grande assurance dans les lectures d'études [articles scientifiques]. » [BD].

L'augmentation de l'efficacité personnelle des étudiantes peut s'expliquer par les nombreuses compétences acquises. Ces compétences ont été développées en regard de six domaines particuliers soit I) la compréhension de la nature des données probantes, 2) la recherche informationnelle, 3) l'analyse d'un article scientifique, 4) l'acquisition de connaissances liées à un domaine précis, 5) l'acquisition d'habiletés communicationnelles, et 6) la maîtrise de la langue seconde.

\section{Compréhension de la nature des données probantes}

Le cours «Processus de la recherche en sciences infirmières 》 est le premier cours du cursus universitaire des étudiantes qui permet de les mettre en contact avec l'environnement de la recherche. Pour plusieurs de celles-ci, la notion de données probantes était inconnue jusqu'à maintenant : « J'ai découvert ce qu'est des données probantes. » [C.]. Comme l'exposent Sylvain et Ouelle, l'utilisation des données probantes n'est pas chose usuelle dans les milieux cliniques (3I). Selon une étude réalisée par Pavikoff et al., près de la moitié des infirmières ne sont pas familières avec la pratique basée sur les données probantes (32).

\section{Recherche informationnelle}

L'identification d'une question PICO a conduit les étudiantes à aller chercher une information précise.
Pour répondre à cette question, l'étudiante a acquis les connaissances permettant l'obtention d'informations par les stratégies de recherche dans les banques de données. Ces connaissances ont été mises en pratique afin de supporter le développement des habiletés informationnelles et technologiques. Le témoignage suivant soutient ce point : « Dorénavant, lorsque j'aurai un questionnement par rapport à ma pratique, je saurai où et comment chercher. » [CD].

\section{Analyse d'un article scientifique}

L'analyse d'un article scientifique repose sur plusieurs connaissances. Pour y arriver, l'étudiante se devait d'acquérir des connaissances sur la structure d'un article scientifique ainsi que de s'approprier la terminologie scientifique, et ce, pour chaque type de devis de recherche. Ces connaissances additionnées ont permis de développer une compréhension pratique de la méthodologie de recherche (but, devis, échantillon, collecte de données, analyses des données et l'interprétation des résultats). Ces connaissances ont permis à l'étudiante de porter un jugement sur la valeur de l'article et des résultats pour sa pratique. Par exemple, une étudiante a rapporté : « J'ai appris comment ceux-ci [les articles scientifiques] étaient faits, ce qui m'a permis de les comprendre. Par exemple, quel type de recherche c'est ? Le devis. » [BG].

D'autres étudiantes ont soulevé leur compétence à juger de la qualité d'un article: « Je sais maintenant critiquer un article scientifique sur sa valeur réelle. Même sans avoir recours à une grille, les termes, devis, échantillonnage, saturation ont moins de secret pour moi. Je les maîtrise. ॥ [AG]. « Je suis maintenant en mesure de voir quel article est fiable et sur lequel je peux me fier pour ma pratique. » [AG].

\section{Acquisition de connaissances liées à un domaine précis}

La structure du club de lecture, en sous-groupes en fonction de différents milieux de pratique, permettait aux étudiantes d'approfondir leurs connaissances sur un sujet particulier. Par exemple, dans le domaine de la santé mentale, différentes problématiques ont été explorées : les méthodes alternatives pour la gestion des symptômes du trouble déficitaire de l'attention avec hyperactivité (TDAH) chez les enfants, les stratégies de gestion de la détresse psychologique chez les personnes âgées et les moyens alternatifs aux contentions physiques. Une étudiante en témoigne ainsi : «Elle [la participation au club de lecture] nous a permis de prendre connaissance de plusieurs articles scientifiques parce que toute seule, je n'aurais pas réussi à lire autant d'articles. » [CF]. Une autre a déclaré : « De plus, j'apprenais aussi sur ce que les autres apportaient. » [BA].

\section{Acquisition d'habiletés communicationnelles}

Les étudiantes ont mentionné que la présentation d'un article scientifique exigeait un certain niveau de maîtrise du contenu et de la terminologie pour être en mesure de critiquer celui- 
ci devant l'animateur et leurs collègues. Cette préparation importante a permis, par ricochet, le développement d'habiletés communicationnelles. Les deux extraits suivants en témoignent : «Pour participer au club de lecture, je devais bien maîtriser le contenu de mon article. » [BA]. «J'ai plus de facilité à verbaliser une recherche. ॥ [BB]. Ces compétences s'avèrent pertinentes dans le contexte où il est reconnu que les habiletés à communiquer, à partager les connaissances ainsi qu'à établir et à maintenir une relation de soutien entre les collègues sont des éléments qui facilitent l'implantation des résultats probants dans la pratique (33).

\section{Maîtrise de la langue anglaise}

Malgré que l'anglais fût souvent mentionné par les étudiantes comme un obstacle pour lire les articles scientifiques trouvés au cours du trimestre, les étudiantes ont affirmé avoir développé des compétences linguistiques à l'égard de la lecture et la compréhension d'articles scientifiques en anglais. Deux d'entre elles se sont exprimées ainsi : « J'ai même appris à traduire les articles en anglais, sans Google, sans que cela me fasse peur. » [AG]. "Cela m'a apporté une meilleure maîtrise de l'anglais. " [D.]. Dans cette étude, la mise en place d'une ressource pour aider les étudiantes a semblé avoir contribué à lever la barrière de la langue anglaise. II apparaît donc que cette barrière peut s'amenuiser, possiblement, comme le décrivent Gagnon et al. (20l I), par le développement de stratégies ou d'automatismes qui enlèvent l'appréhension de la lecture d'articles scientifiques en anglais (34).

\section{Question 2. Rôle des clubs de lecture dans la compréhension des écrits scientifiques}

Les étudiantes qui ont participé au club de lecture affirment que cette méthode pédagogique a facilité l'intégration de la théorie en leur donnant l'occasion d'appliquer une matière théorique pouvant être abstraite pour des néophytes. Le témoignage suivant est éloquent de cette observation : « La pratique surpasse souvent la théorie. Je crois que nous sommes plus habiles à lire et critiquer des articles scientifiques que si on n'avait eu que de la théorie. » [AC].

Les étudiantes ont expliqué que le développement de leur compréhension des écrits scientifiques a reposé sur la combinaison de diverses stratégies soit : I) les expériences d'analyse critique, 2) l'observation de leurs pairs étudiantes et 3) les rétroactions et les échanges. Les stratégies rapportées par les étudiantes correspondent aux sources d'efficacité personnelle décrites par Bandura, soit l'expérience de maîtrise, les expériences vicariantes et la rétroaction (35).

\section{Expériences d'analyse critique}

Les étudiantes ont rapporté que l'action répétée à quatre reprises de devoir se préparer pour présenter les articles lors du club de lecture leur permettait de vivre plusieurs expériences d'analyse critique afin d'être en mesure de la maîtriser. Pour la préparation requise, une étudiante a souligné : «Le nombre d'heures de travail passées à la maison pour préparer le club de lecture. » [BB]. Pour l'effet de la répétition de l'expérience, une autre a rapporté : « L'obligation d'en faire quatre m'a permis de mieux comprendre à chaque fois comment fonctionne un écrit scientifique. ॥ $[\mathrm{BC}]$. Selon Bandura, l'efficacité personnelle peut être augmentée par les expériences actives de maîtrise. Celles-ci se rapportent aux expériences de succès déjà obtenues dans la réalisation d'une tâche et servent d'indicateurs de réussite (35).

\section{Observation des pairs}

Les étudiantes ont affirmé que l'observation de la performance de leurs pairs au cours du club de lecture leur avait permis d'assimiler la théorie. Participer à l'exposé des collègues étudiantes leur a permis de savoir comment elles avaient traité l'analyse critique et la présentation de l'article scientifique. De plus, il semble que l'appropriation du contenu théorique et sa vulgarisation par les autres étudiantes aient permis une meilleure compréhension de la matière du cours grâce à l'usage d'un vocabulaire plus accessible pour les étudiantes. Une étudiante l'a décrit ainsi : «En entendant les autres expliquer, je l'assimile mieux quand c'est vulgarisé par mes collègues. » [.G].

II semble qu'un autre type d'observation ait contribué à améliorer la compréhension des écrits scientifiques. II s'agit de l'observation des grilles d'analyse fournies par la professeure. En l'absence d'un modèle de rôle lorsqu'elles devaient préparer leur analyse individuellement à la maison, les étudiantes ont reconnu l'apport de l'observation des grilles pour accompagner l'analyse des écrits scientifiques. Deux étudiantes ont ainsi exprimé : « En remplissant les grilles, cela m'a permis de bien analyser les articles et de mieux les comprendre. » [CD]. « En répondant aux questionnaires [grilles d'analyse] (étude quantitative, méta-analyse), je pouvais mieux comprendre si mon article était pertinent. ॥ [BB]. L'utilisation de feuilles de travail ou de grilles d'analyse apparaît donc un élément essentiel dans la démarche des étudiantes tel que le suggéraient certaines sources $(4,12,18)$.

En lien avec le concept d'efficacité personnelle de Bandura, cet auteur propose également que celle-ci puisse être améliorée par les expériences vicariantes (modelage par observation), en prenant connaissance d'expériences réalisées par d'autres personnes réalisant le comportement à acquérir tel que cela apparaît avoir été décrit par les participantes (35).

\section{Rétroaction et échanges}

La rétroaction survenant lors du club de lecture a également eu un rôle sur la compréhension des articles scientifiques 


\section{Utilisation d'un club de lecture comme méthode pédagogique pour favoriser l'appropriation des données probantes par des étudiantes au baccalauréat en sciences infirmières}

évalués. Deux sources de rétroaction ont été identifiées par les étudiantes, soit la rétroaction de leurs pairs et celle des responsables. La rétroaction par les autres étudiantes pouvait être un partage de leurs apprentissages, un renforcement positif ou encore des échanges visant à pousser la réflexion de l'étudiante présentatrice. II ressort également l'importance des qualifications des responsables de sous-groupes pour guider les étudiantes dans l'analyse des écrits scientifiques. Les deux extraits suivants soulèvent très bien ce point : «Le feedback reçu à la suite de nos présentations. Les questions des pairs et de l'animateur et la possibilité de discuter avec tous de nos articles étaient très constructives. » $[\mathrm{AA}]$. « En discutant avec les animateurs, en se faisant poser des questions, cela nous amène à réfléchir davantage sur certains points et par le fait même à les comprendre. » [.I].

Encore une fois, les réponses des étudiantes apparaissent être en lien avec les sources de développement de l'efficacité personnelle proposées par Bandura en regard de la rétroaction verbale, c'est-à-dire par la rétroaction sociale de performance. Plus la personne qui cherche à convaincre est crédible, plus le sentiment d'efficacité personnelle sera élevé. Une quatrième source est identifiée par Bandura soit celle des états physiologiques et psychologiques mais cette dernière n'a pas été soulevée par les participants (35).

\section{Question 3. Éléments importants dans l’analyse des écrits scientifiques}

Les étudiantes ont révélé plusieurs éléments importants dans l'analyse critique des écrits scientifiques en vue d'une pratique basée sur les données probantes. En fait, elles ont reconnu que pour utiliser les données probantes dans leur pratique, elles doivent auparavant faire l'analyse de la source. II est intéressant de les voir s'exprimer ainsi : « II ne faut pas se fier à tous les articles. Ils ne sont pas nécessairement tous fiables et représentatifs pour apporter des modifications à notre pratique. » [CD]. « Les écrits ne sont pas tous bons et valides. II est important de bien les analyser pour s'assurer qu'ils peuvent être pertinents pour notre pratique. ॥ [CF]. Les éléments importants à analyser décrits par les étudiantes apparaissent être : I) la formulation de la question PICO, 2) les sources de données et 3 ) l'évaluation de la qualité des écrits scientifiques.

Les étudiantes affirment que pour être en mesure de bien analyser les écrits, elles doivent être habilitées à aller chercher la bonne information pour un problème particulier par la formulation adéquate d'une question PICO. Cela permet notamment d'évaluer le but de l'étude et son rationnel. Une étudiante a ainsi rapporté : « La formulation d'une question de recherche [est importante] pour identifier le but et le rationnel de l'étude. ॥ [.E]. Après avoir formulé la question $\mathrm{PICO}$, les étudiantes ont rapporté l'importance de la fiabilité des sources de données. Par exemple, l'une d'entre elles a mentionné : « II faut faire attention à la revue ou l'article scientifique que nous trouvons, parce que parfois les sources ne sont pas fiables, donc il faut toujours aller valider en regardant les références et la méthode. » $[\mathrm{CH}]$.

Finalement, les étudiantes ont décrit l'importance de l'évaluation de la qualité scientifique en vue de l'application des résultats dans la pratique. Le tableau I expose les éléments méthodologiques à évaluer pour une prise de décision éclairée quant à la transférabilité des résultats tels que rapportés dans les réponses des étudiantes.

\section{Question 4. Application des nouvelles connaissances dans la pratique clinique}

Les étudiantes ont démontré un intérêt à appliquer les connaissances nouvellement acquises dans leur pratique clinique future. Déjà, elles ont identifié quelques moyens pour y parvenir soit : I) se questionner et aller chercher l'information ; 2) lire des articles scientifiques et finalement 3) transférer leurs connaissances dans leur milieu de pratique. Les étudiantes manifestent le désir de poursuivre leur questionnement, d'une part en regard de leur pratique actuelle et, d'autre part, en regard d'un problème clinique particulier. Elles ont pris conscience qu'elles devront toujours questionner leur pratique et ne pas l'asseoir définitivement sur une pratique acquise. Les quatre extraits suivants en témoignent : « En faisant des recherches sur des sujets afin d'apporter des changements ou des prises de conscience. » [.I]. « Je crois que lorsque je vais avoir des questionnements par rapport à une pratique particulière, je vais aller vérifier s'il n'y a pas une recherche qui a été faite à ce sujet. » $[\mathrm{CH}]$. « Je vais chercher davantage les données probantes dans mon domaine de travail pour avoir des soins optimaux. » [.G]. Même les étudiantes du cheminement de formation initiale du cours ont pu constater comme ce cours allait les aider lorsqu'elles se retrouveront sur le marché du travail comme en témoigne l'extrait suivant : «Quand j'aurai des questionnements sur ma pratique, je pourrai répondre à ceux-ci en cherchant des données probantes. » [CG].

Les étudiantes ont manifesté leur motivation à poursuivre leur lecture d'écrits scientifiques dans les réponses suivantes: " Je vais tenter de rester à jour dans les écrits scientifiques. » [BD]. « Je compte lire des articles scientifiques en lien avec mon champ de pratique si je remarque qu'il y a une lacune avec ma pratique infirmière. » [CF]. « Cela m’a donné le goût de continuer à consulter des articles scientifiques. » [AS].

Pour d'autres étudiantes, l'application des nouvelles connaissances se fera dans la pratique elle-même, si l'occasion se présente. Par exemple, deux d'entre elles ont exprimé : « La seule façon que je vois d'appliquer ces notions dans ma pratique est que lorsque je lis un document, un article, de bien regarder certains détails pour m'assurer que celui-ci est applicable à notre milieu et pertinent. » (2). « J'appliquerai 


\begin{tabular}{|c|c|}
\hline Devis utilisé & $\begin{array}{l}\text { «Être capable... de comprendre le devis et la méthode utilisée. » (.C) } \\
\text { «Qu’il existe divers types d'articles scientifiques, par exemple le quantitatif vs qualitatif. » (BF) }\end{array}$ \\
\hline Échantillon & $\begin{array}{l}\text { «Le processus d'échantillonnage pour moi est très important pour la valeur de l'étude, la } \\
\text { taille et le choix de l'échantillonnage. » (AG) }\end{array}$ \\
\hline Résultats & $\begin{array}{l}\text { «La bonne compréhension des résultats. Être capable de reconnaître leur signification. » (AC) } \\
\text { «C'est que les résultats peuvent parfois être significatifs pour que l'on puisse effectuer } \\
\text { un changement dans la pratique infirmière alors que d'autres fois, les recherches n'ont } \\
\text { pas nécessairement été concluantes. » (D.) } \\
\text { «Qu'il y a des articles qui sont incomplets et qu'on ne doit pas prendre leurs résultats pour du } \\
\text { « cash ». On doit avoir une conscience en un jugement pour bien interpréter les résultats.» (.G) }\end{array}$ \\
\hline Critères de rigueur scientifique & $\begin{array}{l}\text { «Pour croire en la validité d'un écrit, il faut d'abord comprendre en quoi il consiste, la } \\
\text { validité et comprendre comment ils se prennent pour s'en assurer.» (C.) }\end{array}$ \\
\hline Forces et limites d'un article scientifique & « Être capable de reconnaître les forces et les limites de l'étude. » (.C) \\
\hline Références & $\begin{array}{l}\text { «Également, je regarde la puissance scientifique des références. » (AG) } \\
\text { «En lisant plusieurs articles, les spécialistes sont souvent nommés. Quand ils ne le sont } \\
\text { pas, nous pouvons nous poser des questions quant à la validité de ces écrits. » (.H) }\end{array}$ \\
\hline
\end{tabular}

Tableau 1.

Éléments méthodologiques importants à considérer dans l'analyse d'un écrit scientifique tels que rapportés par les étudiantes participantes

mes connaissances dans toutes les occasions nécessitant des données fiables et probantes. ॥ [AA]. Également, d'autres étudiantes se voient comme des agents catalyseurs par leur désir de transférer leurs nouvelles connaissances par la discussion avec leurs supérieurs ou leurs collègues $(10,36)$. Cette affirmation est relatée dans les extraits suivants : « En apportant l'idée à mon groupe de travail et en répétant l'expérience avec elle. Leur souligner l'importance de se mettre à jour continuellement pour avoir des compétences optimales. » [BB]. « J'aimerais, lorsque je vais travailler sur une unité dans quelques années et que je vais être familière avec le milieu, me poser des questions et chercher des données probantes pour ensuite en faire profiter mes collègues. ॥ [BG].

Sylvain et Ouellet affirment que malgré les efforts de dissémination des résultats de recherche par les chercheurs, il persiste un écart entre la recherche, la théorie et la pratique infirmière (3I). Une stratégie proposée à l'intégration de la recherche dans la pratique professionnelle est la pratique basée sur les données probantes. Les résultats de la présente étude pourraient indiquer une piste importante pour diminuer cet écart entre la recherche et la pratique puisque plusieurs étudiantes semblent être prêtes à promouvoir le changement et le partage entre les collègues. Ces facteurs ont été identifiés comme des facteurs facilitant pour éventuellement pouvoir agir comme des agents catalyseurs dans les milieux cliniques (I2).

\section{Question 5. Éléments à améliorer}

Les étudiantes ont soulevé divers aspects qui pourraient être améliorés dans l'organisation des clubs de lecture. Premièrement, certaines auraient apprécié pouvoir changer de groupe de club de lecture à quelques occasions pour pouvoir étendre leur champ d'apprentissage. Deuxièmement, au niveau de la structure du cours, il a été suggéré de faire une alternance entre les cours théoriques et les cours dédiés au club de lecture, notamment par la subdivision des types de devis. Troisièmement, pour les stratégies d'enseignement, il a été suggéré de faire une démonstration en classe d'une analyse critique en format club de lecture et de faire un retour en groupe sur la question PICO de chaque étudiante à la dernière séance. Dernièrement, au niveau des responsables de l'animation des sous-groupes, il a été suggéré d'avoir une stabilité au sein de chaque sous-groupe de travail et aussi d'assurer un niveau de compétence uniforme des animateurs. À ce sujet, les étudiantes graduées constituent d'excellentes ressources pour agir comme responsables de sous-groupes tel que cela a été proposé antérieurement (20).

Bien que les questionnaires ne posaient pas de questions directement sur les obstacles rencontrés, certains ont été relevés à l'intérieur des réponses des étudiantes. L'obstacle majeur identifié par ces dernières fut la langue anglaise. « $\mathrm{Ce}$ que j'ai trouvé le plus difficile était les articles en anglais. » 


\section{Utilisation d'un club de lecture comme méthode pédagogique pour favoriser l'appropriation des données probantes par des étudiantes au baccalauréat en sciences infirmières}

[BF]. « L'anglais qui était un obstacle pour bien analyser les textes scientifiques. ॥ $[\mathrm{CC}]$. Un deuxième obstacle est celui de l'expertise acquise qui demeure encore précaire. «Par contre, je ne suis pas une experte et je sais que j'ai encore beaucoup à apprendre. » $[\mathrm{CH}]$. Polit et Beck ont présenté une classification des obstacles ou facilitateurs au transfert des résultats de recherche (37). Elles les ont classés dans trois catégories soit ceux liés à la recherche en soi, ceux liés aux caractéristiques des professionnels de la santé et ceux liés à l'organisation. Les deux obstacles observés dans cette étude correspondent aux obstacles liés aux caractéristiques des professionnels puisqu'ils font appel aux capacités de l'étudiante à lire des articles en anglais et à un besoin de connaissance supplémentaire.

\section{Limites de l'étude}

La présente étude comporte certaines limites, notamment en ce qui concerne l'échantillon, la méthode de collecte des données par questionnaire ainsi que la position de la professeure qui était également responsable de la recherche. Pour l'échantillon, il s'est avéré que toutes les étudiantes participantes n'ont pas complété les questionnaires. Cependant, il est probable que les réponses des non participantes auraient été dans le même sens que celles des répondantes puisque cette non participation est liée à l'organisation du cours plutôt qu'à une volonté de ne plus participer. En ce qui a trait au questionnaire, il a été observé au cours des analyses que certaines réponses étaient peu approfondies. L'utilisation d'entrevues, bien que difficilement réalisable auprès d'un tel échantillon, aurait permis d'aller davantage en profondeur dans les réponses données. Enfin, malgré toutes les précautions prises pour assurer la confidentialité de la participation et des réponses des étudiantes, il est possible que les réponses aient été influencées par le fait que la professeure du cours était responsable de la recherche.

\section{CONCLUSION}

Cette étude apparaît être la première à décrire en profondeur l'appréciation et les effets de la participation à un club de lecture obligatoire à l'intérieur d'un cours de recherche en sciences infirmières universitaire. Les étudiantes ont soutenu une appréciation globale très positive. Les résultats ont permis de décrire les différentes compétences acquises par les étudiantes ainsi que différents mécanismes inspirés de la théorie de l'efficacité personnelle de Bandura ayant soutenu le développement de ces compétences (35). II apparaît clairement que la méthode pédagogique des clubs de lecture devrait être valorisée et implanter à plus large échelle. Comme le soulignaient Melnyk et al. (36), la recherche doit prendre vie pour les étudiantes de premier cycle afin qu'elles puissent comprendre comment elle peut être utilisée pour améliorer les pratiques et conduire aux meilleurs résultats pour les patients et leurs familles. Ces étudiantes doivent être exposées à cette littérature pour appuyer les décisions futures qu'elles prendront dans leurs milieux cliniques et la participation à un club de lecture est sans contredit un premier pas dans cette direction.

Cette démonstration devrait ainsi être poursuivie dans d'autres milieux, avec des ajustements au niveau de la structure du cours. Il faudrait également se pencher sur des stratégies permettant le maintien des compétences développées lors de la participation au club de lecture à l'intérieur du cours de recherche dans la suite du cursus universitaire ainsi que dans la carrière future des étudiantes. La mise en place d'une pratique basée sur les données probantes est un processus d'amélioration continue de la pratique. Pour soutenir ce processus, il incombe de mettre en place un continuum d'apprentissage de la pratique basée sur les données probantes dans les différents milieux cliniques où se trouvent les infirmières.

\section{FINANCEMENTS}

Ce projet a pu être réalisé grâce au soutien financier du département des sciences de la santé (pour l'embauche des responsables des sous-groupes), du programme Opération réussite (pour le soutien à la lecture de l'anglais) et du décanat des études de premier cycle de l'UQAC (pour la collecte et l'analyse des données).

\section{CONFLITS D'INTÉRÊTS}

Aucun des auteurs ne déclare de conflits d'intérêts.

\section{Références bibliographiques}

1. Scott K, Mcsherry R. Evidence-based nursing : Clarifying the concepts for nurses in practice. J Clin Nurs. 2009;(18):1085-95.

2. Kleinpell RM. Rediscovering the value of the journal club. Am J Crit Care. 2002;(11):412-14.

3. Institute of medicine. Health professions education : A bridge to quality. National Academies Press;2003.

4. Steenbeek $A$, et al. Using an interactive journal club to enhance nursing research knowledge acquisition, appraisal, and application. Int J Nurs Educ Scholarsh.2009;(6):1p.

5. Flesner MK, et al. Introduction to evidence-based practice in nursing and health care. J Prof Nurs. 2010;(26):65-85.

6. Odell E, Barta K. Teaching evidence-based practice : The bachelor of Science in nursing essentials at work at the bedside. J Prof Nurs. 2011;(27):370-77.

7. Ordre des infirmières et infirmiers du Québec. La relève infirmière: une profession, une formation;2012.

8. Halcomb EJ, Peters K. Nursingstudent feedback on undergraduate research education : Implications for teaching and learning. Contemp Nurse. 2009;(33):59-68. 
9. Mccurry MK, Martins DC. Teaching undergraduate nursing research : A comparison of traditional and innovative approaches for success with millennial learners. J Nurs Educ. 2010;(49):276-79.

10. Moch SD, Cronje RJ, Branson J. Part 1. Undergraduate nursing evidence-based practice education : Envisioning the role of students. J Prof Nurs. 2010;(26):5-13.

11. Patel PC, et al. Evidence-based practice and a nursing journal club : An equation for positive patient outcomes and nursing empowerment. J Nurs Staff Dev. 2011;(27):227-30.

12. Thompson CJ. Fostering skills for evidence-based practice: The student journal club. Nurse Education in Practice. Nurse Educ Pract. 2006;(6):69-77.

13. Kim SC, et al. Evidence-based practice-focused interactive teaching strategy : A controlled study. J Adv Nurs. 2009;(65):1218-27.

14. Moore SL. Incorporating a journal club in associate degree clinical conferences. Teach Lear Nurs. 2009;(4):122-27.

15. Khan KS, Gee H. A new approach to teaching and learning in journal club. Med Teach. 1999;(21):289-93.

16. Glazer FS. Journal clubs - A successful vehicle to science literacy. J Coll Sci Teach. 2000;(29):320-24.

17. Marcil I, Goulet M. Le journal club : de la recherche à la pratique. Infirm Que. 2002;(9):46-51.

18. Goodfellow LM. Can a journal club bridge the gap between research and practice ? Nurse Educ. 2004;(29):107)10.

19. Laaksonen C, et al. Journal club as a method for nurses and nursing students' collaborative learning : a descriptive study. Health Sci J. 2013;(7):285-92.

20. Missal B, et al. A university and health care organization partnership to prepare nurses for evidence-based practice. J Nurs Educ. 2010;(49):456-61.

21. Mattila LR, et al. Journal club intervention in promoting evidencebased nursing : Perceptions of nursing students. Nurse Educ Pract. 2013;(13):423-28.

22. Williams AF. Free-style PBL using a journal club. Australian Electronic J Nurs Educ. 2002;(8):9p.

23. Marleau D, Lapointe J. Portrait de la relève infirmière : 2012-
2013, Ordre des infirmières et infirmiers du Québec ; 2013.

24. Grove SK, Burns N, Gray JR. The practice of nursing research. Elsevier;2013.

25. Stillwell SB, et al. Asking the clinical question : A key step in evidence-based practice. Am J Nurs. 2010;(110):58-61.

26. Davies B, Logan J. Lire des textes de recherche. Elsevier Mosby.2010.

27. Fortin $\mathrm{M}$, et al. La pratique basée sur les données probantes \& analyse critique de la littérature médicale. Unité de médecine de famille du CSSS de Chicoutimi;2012.

28. Benhaberou-Brun D. Les pratiques exemplaires : Le défi de I'infirmière au quotidien. Perspect Infirm. 2007;(4):35-37.

29. Miles MB, Hubermann AM. Qualitative Data Analysis : A Methods Sourcebook. Thousand Oeks, CA;2013.

30. Conseil International des Infirmières. Combler l'écart : Des preuves aux actes ; 2012.

31. Sylvain H, Ouellet N. In : Profession infirmière : Quelle place et quelles pratiques pour l'avenir? Delmas and C. Sliwka ; 2009.

32. Pravikoff DS, Pierce ST, Tanner A. Evidence-based practice readiness study supported by academy nursing informatics expert panel. Nurs Outlook. 2005;(53):49-50.

33. Stetler CB, et al. Role of "external facilitation" in implementation of research findings : A qualitative evaluation of facilitation experiences in the Veterans Health Administration. Implement Sci. 2006;(1):23-23.

34. Gagnon J, et al. La pratique infirmière informée par des résultats de recherche : La formation de leaders dans les organisations de santé, une avenue prometteuse. Rech Soins Infirm. 2011;(105):76-82.

35. Bandura A. Auto-efficacité : Le sentiment d'efficacité personnelle. Paris: De Boeck Université;2007.

36. Melnyk BM, et al. Evidence-based practice. Evidence-based practice: the past, the present, and recommendations for the millennium. Pediatr Nurs. 2000;(26):77-80.

37. Polit DF, Beck CT. Nursing research : Generating and assessing evidence for nursing practice. Wolters Kluwer/Lippincott Williams \& Wilkins ; 2008. 